\title{
Comparison of the Short Term Interest Rate Models: Parametric versus Non parametric Approach
}

\author{
Mouna Ben Salah ${ }^{1}$
}

\begin{abstract}
This article attempts to identify the best features the short term interest rates stochastic process.

We studied nine different linear models of short term interest rates. The choice of these models was the aim of analyzing the relevance of certain specifications of the short term interest rate stochastic process, the effect of mean reversion and the sensitivity of the volatility to the level of interest rate.

We studied also the relevance of the Ait-Sahalia (1996b) nonlinear interest rate model.

To further study the accurate parametric specification of the interest rate stochastic process we used a nonparametric estimation of the drift and the diffusion functions.

The yield on three months treasury bills is used as a proxy for the short term interest rates. The parameters of the different linear stochastic process are estimated using the generalized method of moments. A semi parametric approach is used to estimate the non linear Ait Sahalia model (1996b). The kernel regression is used as a nonparametric approach to estimate the interest rate process.

The results show that the effect of mean reversion is not statistically significant and that volatility is highly sensitive to the level of interest rates. The results prove also that both the drift and the diffusion functions should be nonlinear and that the nonlinear specification proposed by Ait Sahalia (1996b) model is not accurate.
\end{abstract}

JEL classification numbers: C13, C14, C15, C22, C32, C52, E43, E47.

Keywords: short term interest rate, diffusion process, GMM, nonlinear, maximum likelihood, nonparametric, Monte Carlo simulation.

\footnotetext{
${ }^{1}$ National School of Computer Sciences, Campus Universitaire de la Manouba, Manouba 2010, Tunisia.
}

Article Info: Received : February 10, 2014. Revised : March 9, 2015.

Published online : June 1, 2015 


\section{Introduction}

Understanding the short term interest rate stochastic behaviour is very important for a wide range of applications. Such as the conduct of monetary policy, the financing of public debt, estimating the expectations of real economic growth and inflation and determining prices in financial market. Several models have been developed to explain the stochastic process of the short term interest rate in a continuous-time framework. A list of these models include linear models, those by Merton (1973), Vasicek (1979), Brennan and Schwartz (1980a), Dothan (1978), Cox, Ingersoll and Ross (1980, 1985b), Rendleman and Bartter (1980), Cox and Ross (1976) and Chan, Karoly, Longstaff and Schwartz (1992) and the nonlinear Ait-Sahalia (1996b) model. These models make the assumption that the short term interest rate follows a gauss-wiener process. The process of the short term interest rate, $r$, has the following formulation:

$\mathrm{dr}=\mu(\mathrm{r}) \mathrm{dt}+\sigma(\mathrm{r}) \mathrm{dw}$

The drift rate, $\mu$, and the instantaneous standard deviation, $\sigma$, are functions of $r$, but independent of time, and $\mathrm{w}$ is a wiener process.

The models mentioned above differ by their specifications of the drift and the diffusion function of the short term interest rate process.

One of the key points in this area is if their specification of the interest rate dynamic is correct or not. Is the short-rate drift function linear or nonlinear? Is the short-rate diffusion function constant, linear or nonlinear?

This article aim to present answers to this questions and determine the appropriate features of the short term interest rate process. The study will investigate the shape of the drift and of the diffusion functions using nonparametric estimation. The nonparametric approach avoids making parametric assumptions about both the drift and the diffusion functions and estimates the both from the observed data.

\section{The Data}

We estimate the interest rate models considering as proxy of the short-term interest rate, the US 3 month Treasury bill rate. The data are weekly and cover the period from January 1970 to December 2011, providing 2165 observations. The observations are taken from the Federal Reserve website of Saint Louis.

The time series of short-term interest rates shown in Figure 1 is suggestive of a change in the process during the late 1970 and early 1980. Both the level and the volatility appear elevated.

The table 1 shows the means, standards deviations and part of the 11 autocorrelation of the weekly rates and the weekly changes in the spot rate. The unconditional average level of the weekly rate is $5.46 \%$, with a standard deviation of $3.13 \%$. Although the autocorrelations in interest rate level decays very slowly, those of the week-to week changes are generally small and are not consistently positive or negative. This offers some evidence that the interest rates changes are stationary. The results of a formal augmented Dickey-fuller nonstationarity test are also reported in Table 1. The null hypothesis of nonstationarity is accepted for the interest rate levels but is rejected for the interest rate changes at the $1 \%$ significance level. 
Table 1: Summary statistics of the data and stationarity test

\begin{tabular}{|l|l|l|l|l|l|l|l|l|l|}
\hline \multicolumn{2}{|c|}{ Summary statistics } \\
\hline & $\mathrm{N}$ & Mean & $\begin{array}{l}\text { Standard } \\
\text { deviation }\end{array}$ & $\rho_{1}$ & $\rho_{3}$ & $\rho_{5}$ & $\rho_{7}$ & $\rho_{9}$ & $\rho_{11}$ \\
\hline $\mathrm{r}_{\mathrm{t}}$ & 2165 & 5,46 & 3,13 & 0,995 & 0,983 & 0,969 & 0,955 & 0,941 & 0,928 \\
\hline $\mathrm{r}_{\mathrm{t}+1}-\mathrm{r}_{\mathrm{t}}$ & 2164 & $-0,003$ & 0,22 & 0,262 & 0,056 & 0,052 & - & - & - \\
0
\end{tabular}

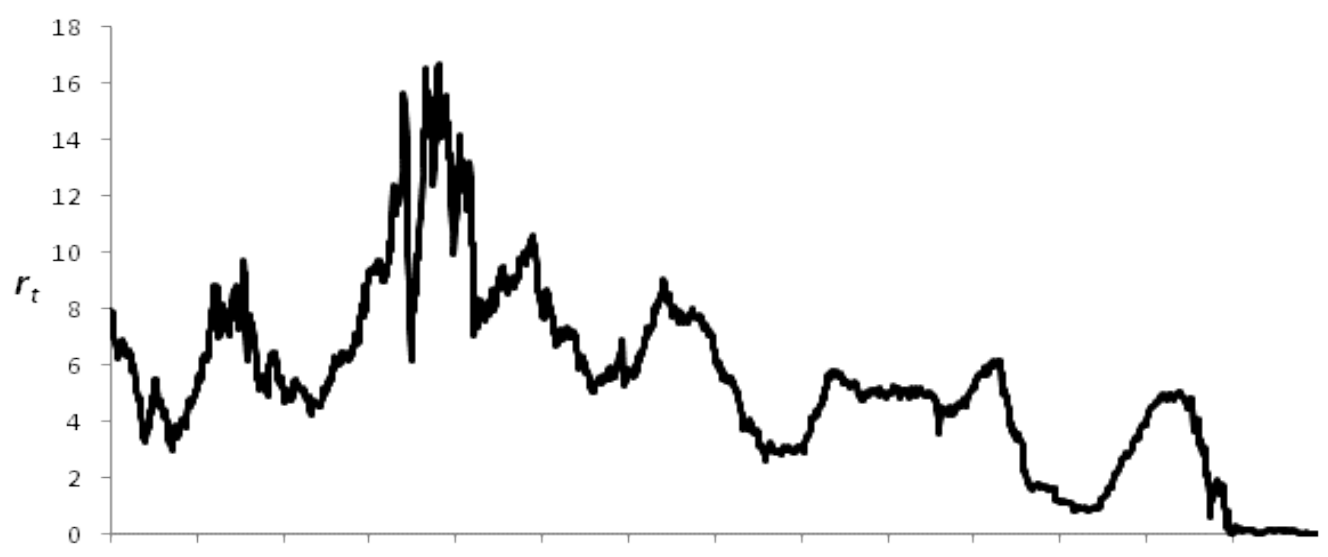

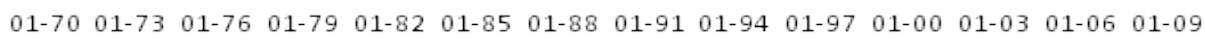

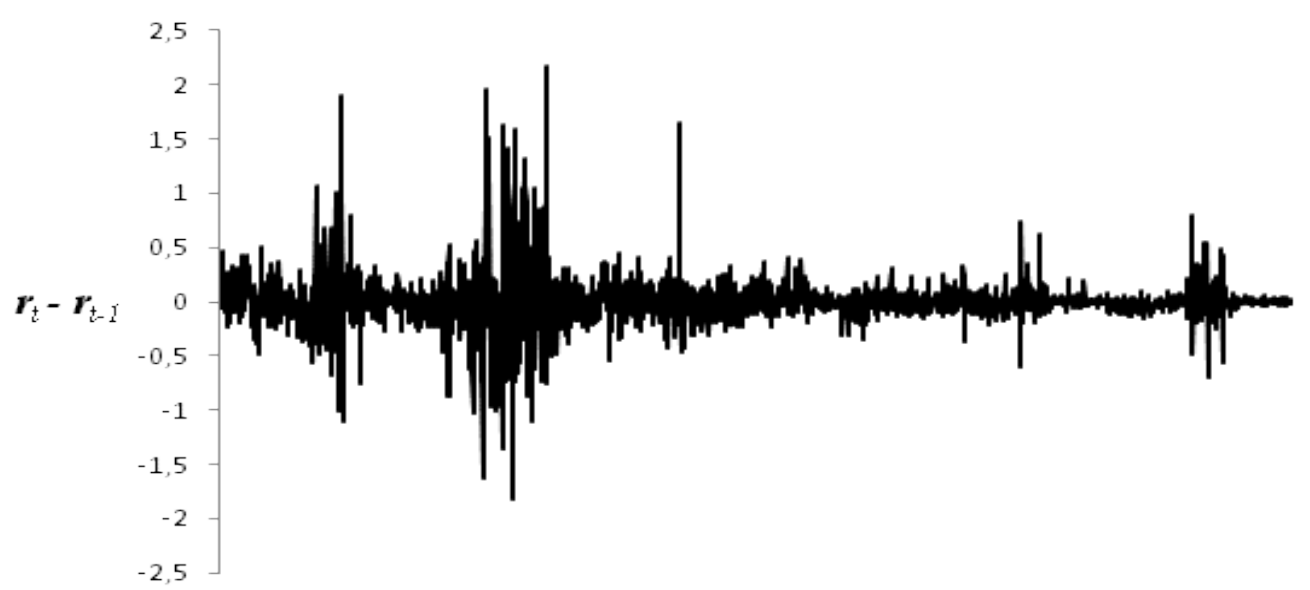

Figure 1: (a) The 3-month T-Bill rate; (b) absolute changes in the 3-month T-Bill rate 


\section{Research Design and Methodology}

\subsection{The Parametric Approach}

\subsubsection{The linear models}

We follow the Chan, Karolyi and Sanders (1992) econometric approach to compare the ability of nine models to capture the stochastic behaviour of the short term interest rate. The authors present a common framework in which different models could be nested. The following stochastic differential equation defines a broad class of interest rate processes,

$$
d r_{t}=\left(a+b r_{t}\right) d t+\sigma r_{t}^{\gamma} d w
$$

The dynamics implies that the conditional mean and variance of changes in the short term interest rate depends on the levels of $r$. The model incorporates mean reversion on the interest rate; i.e, the interest rate is pulled back over time to some long-run average. When $r$ is high, mean reversion tends to cause it to have a negative drift; when $r$ is low mean reversion tends to cause it to have a positive drift. The mean reversion phenomenon is included in the stochastic process by the specification of the drift, $\beta(\alpha-r)$, where the speed of adjustment is given by the parameter $\beta$ and the long-run average is given by the parameter $\alpha$. The short rate is pulled to level $\alpha$ at rate $\beta$. So we have that $a=\alpha \beta$ and $b=$ $-\beta$.

The parameters of the stochastic process given by (1) are estimated in discrete time using Generalized Method of Moments Technique of Hansen (1982). This technique has a number of advantages which makes it one of the best methods for estimating the shortterm interest rate process. Indeed, GMM provides a unified approach to the econometric estimation of all different types of short-term interest rates. Moreover, to achieve the asymptotic convergence of the estimator, GMM does not require that the distribution of the interest rate changes is normal but only stationary and ergodic is to say that the instantaneous conditional residuals variance is proportional to the length of the sample. This feature is of particular importance for the estimation of the short-term interest rate models when each model implies a different distribution of interest rate changes. In fact, for the Merton (1973) and Vasicek (1977) models, changes in interest rates are normal, while for the model Cox Ingersoll and Ross (1985), they are proportional to a noncentral $\chi^{2}$

We test the restrictions imposed by the alternative short term interest rate models nested within equation (1).

Several models can be obtained from (1) placing the appropriate restrictions on the four parameters $\alpha, \beta, \sigma$ and $\gamma$. The specifications that we focus on are presented in Table 2: 
Table 2: Alternative models of short-term interest rate and parameter restrictions imposed

\begin{tabular}{lcccc}
\hline & \multicolumn{4}{c}{ Restrictions } \\
\cline { 2 - 5 } Model & $\mathrm{a}$ & $\mathrm{b}$ & $\sigma$ & $\gamma$ \\
\hline Chan, Karoly, Longstaff \& Sanders (1992) & - & - & - & - \\
Merton (1973) & - & 0 & - & 0 \\
Vasicek (1977) & - & - & - & 0 \\
Cox, Ingersoll \& Ross (1985) & - & - & - & 0.5 \\
Dothan (1978) & 0 & 0 & - & 1 \\
Rendleman \& Bartter (1980) & 0 & - & - & 1 \\
Brennan \& Schwartz (1980) & - & - & - & 1 \\
Cox, Ingersoll \& Ross (1980) & 0 & 0 & - & 1.5 \\
Cox \& Ross (1976) & 0 & - & - & -
\end{tabular}

We estimate the parameters of the continuous-time model using a discrete-time econometric specification

$$
\begin{aligned}
& r_{t+1}-r_{t}=a+b r_{t}+\varepsilon_{t+1} \\
& E\left[\varepsilon_{t+1}\right]=0, E\left[\varepsilon_{t+1}^{2}\right]=\sigma^{2} r_{t}^{2 \gamma}
\end{aligned}
$$

This discrete-time model has the advantage of allowing the variance of interest rate changes to depend directly on the level of the interest rate in a way consistent with the continuous-time model.

Define $\theta$ to be the parameter vector with elements $\alpha, \beta, \sigma^{2}$ and $\gamma$ and given $\varepsilon_{t+1}=r_{t+1}-r_{t}-a-b r_{t}$, estimators of these parameters are obtained from the first and second moments conditions. We define also two instrumental variables, a constant and $r_{t}$. we obtain then, four orthogonality restrictions.

$$
\mathrm{f}_{\mathrm{t}}\left(\mathrm{a}, \mathrm{b}, \sigma^{2}, \gamma\right)=\left[\begin{array}{c}
\varepsilon_{\mathrm{t}+1} \\
\varepsilon_{\mathrm{t}+1}^{2}-\sigma^{2} \mathrm{r}_{\mathrm{t}}^{2}
\end{array}\right] \otimes\left[\begin{array}{c}
1 \\
\mathrm{r}_{\mathrm{t}}
\end{array}\right]=\left[\begin{array}{c}
\varepsilon_{\mathrm{t}+1} \\
\varepsilon_{\mathrm{t}+1} \mathrm{r}_{\mathrm{t}} \\
\varepsilon_{\mathrm{t}+1}^{2}-\sigma^{2} \mathrm{r}_{\mathrm{t}}^{2 \gamma} \\
\left(\varepsilon_{\mathrm{t}+1}^{2}-\sigma^{2} \mathrm{r}_{\mathrm{t}}^{2 \gamma}\right) \mathrm{r}_{\mathrm{t}}
\end{array}\right]
$$

Under the null hypothesis that the restrictions implied by (2) and (3) are true, $E[f(\theta)]=0$ The GMM procedure consists of replacing $E[f(\theta)]$ with its sample counterpart, $g[\theta]$, using $\mathrm{T}$ observations where

$g(\theta)=\frac{1}{T} \sum_{t=1}^{T} f(\theta)$

And then choosing parameters that minimize the quadratic form,

$$
J_{T}(\theta)=g_{T}^{\prime}(\theta) W_{T}(\theta) g_{T}(\theta)
$$

Where $W_{T}(\theta)$ is a positive-definite symmetric weighting matrix. 
The minimized value of the quadratic form in (6) is distributed $\chi^{2}$ under the null hypothesis that the model is true with degree of freedom equal to the number of orthogonality conditions net of the number of the parameters to be estimated. This $\chi^{2}$ measure provides a goodness-of-fit test for the model. A high value of this statistic means that the model is misspecified.

In addition, in order to gauge further the relative performance of the alternative nested models, we test their forecast power of interest rate changes. In addition, we test their forecast power for squared interest rate changes, which provide simple ex-post measures of interest rate volatility. This is done by first computing the time series of conditional expected-yield changes and conditional variances for each model using the fitted values of (2) and (3). We then compute the proportion of the total variation in the ex post yield changes or squared yield changes that can be explained by the conditional expected-yield changes and conditional volatility measures, respectively. We refer to this as the coefficient of determination, or $\mathrm{R}^{2}$. These $\mathrm{R}^{2}$ values provide information about how well each model is able to forecast the future level and volatility of the short term rate. The results are presented in the last two columns of Table 3.

\subsubsection{The non linear model}

Ait Sahalia (1996b) presented a model of the short term interest rate which the drift and the diffusion functions are both nonlinear.

$d r=\left(\propto_{0}+\propto_{1} r_{t}+\propto_{2} r_{t}^{2}+\frac{\alpha_{3}}{r_{t}}\right) d t+\left(\beta_{0}+\beta_{1} r_{t}+\beta_{2} r_{t}^{\beta_{3}}\right) d w$

This nonlinear specification imposes some restrictions on the parameters values.

- $\beta_{0} \geq 0$ (and $\beta_{2}>0$ if $\beta_{0}=0$ and $0<\beta_{3}<1$ or ou $\beta_{1}>0$ if $\beta_{0} 0$ and $\beta_{3}>1$ ), is necessary for the volatility to be positive in the neighborhood of the zero

- $\beta_{2} \geq 0$ if either $\beta_{3}>1$ or $\beta_{1}=1$, and $\beta_{1}>1$ if either $0<\beta_{3}<1$ or $\beta_{2}=0$, is necessary for the volatility to be positive in the neighborhood of the infinity

- $\propto_{2} \leq 0$ and $\propto_{1}<0$ if $\propto_{2}=0$, ensure that the drift is mean reverting at high interest rate values.

- $\propto_{3}>0$ and $2 \propto_{3} \geq \beta_{0} \geq 0$ or $\propto_{3}=0, \propto_{0}>0, \beta_{0}=0, \beta_{3}>1$ and $2 \propto_{0} \geq \beta_{1}>$ 0 ,guarantees that zero is unreached

To estimate this model we have followed the same semi parametric approach used by Ait Sahalia (1996b). The stationary density is nonparametrically estimated based on the kernel function, which is used to estimate the parameter vector $\left(\alpha_{0}, \propto_{1}, \propto_{2}, \propto_{3}, \beta_{0}, \beta_{1}, \beta_{2}, \beta_{3}\right)$ according to the method of maximum likelihood.

Given a sample of T observations, the estimation method is built in three stages.

The first step is to estimate the stationary density using a nonparametric method which is the Gaussian kernel regression as follow:

$\hat{p}(u)=\frac{1}{T h} \sum_{t=1}^{T} K\left(\frac{u-r_{t}^{\Delta}}{h}\right)$ with $\mathrm{K}$ is a Gaussian kernel function of the form $K(u)=$ $\frac{1}{\sqrt{2 \pi}} \exp \left(-\frac{1}{2} u^{2}\right)$ where $\mathrm{h}$ is the smoothing parameter which determines how the neighboring point are taken into account to build the density estimator to $\mathrm{u}$.

The second step is to build an explicit relationship between the stationary density $\mathrm{p}(\mathrm{x})$, the mean and volatility using the Kolmogrov forward equation. In particular, if $\mu(x, \psi)$ and $\sigma(\mathrm{x}, \psi)$ are respectively the functional forms of the drift and the diffusion functions 
with $\psi$ is the vector of parameters $\left(\propto_{0}, \propto_{1}, \propto_{2}, \propto_{3}, \beta_{0}, \beta_{1}, \beta_{2}, \beta_{3}\right)$, then the stationary density of the nonlinear model is then of the form:

$p(x, \psi)=\frac{\xi(\psi)}{\sigma^{2}(x, \psi)} \exp \left\{\int^{x} \frac{2 \mu(u, \psi)}{\sigma^{2}(u, \psi)}\right\}$

Or the lower limit of the integral is arbitrary and $\xi(\psi)$ is a constant that ensures that $\mathrm{p}(\mathrm{x}$, $\psi$ ) integrates to 1 . The basis of the estimation method developed by Ait Sahalia (1996b) is that if the specification of the drift and the diffusion functions are appropriate, then, for the estimated values $\left(\psi^{*}\right)$, the stationary density $\mathrm{p}(\mathrm{x}, \psi)$ is very close to the nonparametric density estimated from the observed data.

In the third step, we estimate the parameters of the drift and the diffusion functions $\left(\alpha_{0}, \propto_{1}, \alpha_{2}, \propto_{3}, \beta_{0}, \beta_{1}, \beta_{2}, \beta_{3}\right)$, so that the stationary density involved by the drift and the diffusion functions is as close as possible to the nonparametric stationary density. The vector of the estimated parameters $(\psi *)$ is chosen such that it minimizes the squared difference between the density of the stationary pattern and the nonparametric one.

Then:

$\psi^{*}=\arg \min \frac{1}{T} \sum_{i=1}^{T}\left(p\left(x_{t}, \psi\right)-p\left(x_{t}\right)^{2}\right.$

This gives the vector of estimated parameters $\left(\psi^{*}\right)=\left(\propto_{0}, \propto_{1}, \propto_{2}, \propto_{3}, \beta_{0}, \beta_{1}, \beta_{2}, \beta_{3}\right)^{*}$.

\subsubsection{The Monte Carlo Simulation study of the interest rate models performance}

To further compare the performance of each model to capture the stochastic evolution of the short term interest rate, we simulate the path of the interest rate produced by each model and we compare it to the real short term interest rate stochastic path.

To generate data from the interest rate model specification, we consider a first order Euler's approximation of the stochastic process of each model.

The study of the predictive performance of the different models will be on both sides, a study of the predictive performance, "in the sample" and "out of the sample".

- The first period "in the sample" cover the period from 1979 to 1982 which is a high volatile period. The purpose of this choice is to study the predictive performance of the models in this exceptional period.

- The second period "in the sample" cover the period from 2007 to 2008 which is the subprime crisis period and at the end of 2008, the Federal Reserve have decide to reduce interest rates at a range of $0 \%$ to $0.25 \%$.

- Contrary to the two first periods, the third "in the sample" period from 1997 to 1998 is relatively a stable period.

- The "out of the sample" period cover the period from 2010 to 2011 , characterized by low interest rates as decided by the Federal Reserve.

The performance of each model to predict the real short term interest rate path is measured by the "Mean Squared Error":

$$
M S E=\frac{1}{N} \sum_{i=1}^{N} \sqrt{r_{i o}-r_{i s}}
$$

Where $N$, is the observation number, $r_{i o}$, is the $\mathrm{i}^{\text {th }}$ observed interest rate and $r_{i s}$, is the $\mathrm{i}^{\text {th }}$ simulated interest rate. 


\subsection{The Nonparametric Approach}

One potentially serious problem with any parametric model that prefers one functional form another is misspecification which can lead to serious pricing and hedging errors.

For further study the short interest rate stochastic process specification we use the nonparametric approach to estimate the functional form of the drift and diffusion functions of the interest rate stochastic process. The nonparametric approach does not impose any restrictions on their functional forms but leave them unspecified. The resulting functional forms should result in a process that follows interest rate closely.

The nonparametric approach presents the flexibility to fit the data allowing the identification of the appropriate specification of the interest rates stochastic process.

Florens-Zmirou (1993) and Ait-Sahalia (1996a) pioneered the idea of modeling the diffusion function of the stochastic interest rate process by the data themselves through a nonparametric approach. The idea has been extended to both the drift and the diffusion functions by Stanton (1997), Jiang and Knight (1997) and, more recently, by Bandi \& Phillips (2003).

Renò, Roma and Schaefer (2006) prove that the Stanton and Bandi \& Phillips estimators perform better than the Ait Sahalia (1996a) estimator.

In this study we follow the Stanton approach (1997). In contrary to the Ait-Sahalia (1996a) that proposes a nonparametric diffusion function estimator based on the linear mean-reverting drift function for the stochastic process, the Stanton approach (1997) avoids making parametric assumptions about either the drift or the diffusion functions of the interest rate stochastic process; it estimates both functions nonparametrically from observed data.

This approach consists of the construction of approximation of the true drift and the diffusion functions then these approximations are estimated nonparametrically from discretely sampled data. More specifically, Stanton (1997) uses the infinitesimal generator and a Taylor series expansion to give the first order approximations to the drift and the diffusion functions.

Consider, the diffusion process of the interest rate, $r_{t}$, which satisfies the stochastic differential equation:

$\operatorname{dr}(\mathrm{t})=\mu\left(\mathrm{r}_{\mathrm{t}}\right) \mathrm{dt}+\sigma\left(\mathrm{r}_{\mathrm{t}}\right) \mathrm{dw}_{\mathrm{t}}$

The first order approximations of the drift and diffusion functions, under the Taylor series is respectively as follows:

$$
\begin{aligned}
& \mu\left(r_{\mathrm{t}}\right)=\frac{1}{\Delta} \mathrm{E}\left(\mathrm{r}_{\mathrm{t}+\Delta}-\mathrm{r}_{\mathrm{t}}\right)+\mathrm{O}(\Delta) \\
& \sigma^{2}\left(\mathrm{r}_{\mathrm{t}}\right)=\frac{1}{\Delta} \mathrm{E}\left(\mathrm{r}_{\mathrm{t}+\Delta}-\mathrm{r}_{\mathrm{t}}\right)^{2}+\mathrm{O}(\Delta)
\end{aligned}
$$

Where $\Delta$ denotes a discrete time step in a sequence of observations of the process $r_{t}$ and $\mathrm{O}(\Delta)$, the asymptotic order symbol where $\lim _{\Delta \rightarrow 0} O(\Delta)=0$.

The nonparametric estimation of the approximations of the drift and the diffusion functions are based on the stationary density. 
Let $\left\{r_{t}^{\Delta}\right\}_{t=1}^{T}$ be a sample of size T from the continuous time process $\mathrm{r}_{\mathrm{t}}$, observed at discrete interval $\Delta$. Furthermore, let $\left\{u_{i}\right\}_{i=1}^{N}$ be a set of size $\mathrm{N}$ points defining an equally spaced partition of a subset of the support of the stationary density. If the stationary density of $r_{t}$ is denoted $f(u)$, the Rosenblatt-Parzen kernel estimator is of the form

$$
\hat{f}(u)=\frac{1}{T h} \sum_{t=1}^{T} K\left(\frac{u-r_{t}}{h}\right)
$$

The kernel estimator is completely characterized by the choice of a particular kernel function and the appropriate bandwidth $h$.

The kernel function provides a method of weigthing "nearby" observations in order to construct a smoothed histogram of the density estimator. In our case we use the Gaussian kernel, $K(u)=\sqrt{2 \pi} e^{-1 / 2 u^{2}}$.

The parameter $\mathrm{h}$ is called the smoothing parameter; it determines the width of the kernel function around any partition point $\mathrm{u}_{\mathrm{t}}$. it specifies how (and how many) "neighboring" points of $r_{t}^{\Delta}$, are to be considered in constructing the density estimator at $r_{t}$. in our case we choice $h=4 \times \hat{\sigma} T^{-1 / 5}$

Now, the drift and diffusion function can estimated nonparametrically using the familiar Nadaraya-watson kernel regression estimator as follow:

$$
\begin{aligned}
& \hat{\mu}(r)=\frac{\sum_{t=1}^{T-1}\left(r_{t+\Delta}-r_{t}\right) K\left(\frac{r-r_{t}}{h}\right)}{\sum_{t=1}^{T-1} K\left(\frac{r-r_{t}}{h}\right)}, \\
& \hat{\sigma}(r)^{2}=\frac{\sum_{t=1}^{T-1}\left(r_{t+\Delta}-r_{t}\right)^{2} K\left(\frac{r-r_{t}}{h}\right)}{\sum_{t=1}^{T-1} K\left(\frac{r-r_{t}}{h}\right)}
\end{aligned}
$$

\section{Empirical Finding and Result Analysis}

In this section, we present our empirical results. We begin by the linear models, the unrestricted and the eight restricted interest rate processes. We present after the estimation results of the nonlinear model. We compare after their ability to reproduce the stochastic path of the short term interest rate through a simulation study. Finally, we present the results of the nonparametric estimation of the drift and diffusion function of the short term interest rate process.

\subsection{Estimation results of linear models}

Table 3 reports the parameters estimates, asymptotic t-statistics, and GMM minimized criterion $\left(\chi^{2}\right)$ values for the unrestricted model and for the each of the eight nested 
models. As shown, the models vary in their explanatory power on interest rate changes. The $\chi^{2}$ tests for goodness-of-fit suggest that all the models that assume $\gamma \leq 1$ are misspecified. In fact Merton (1973), Vasicek (1977), Cox Ingersoll \& Ross (1985), Dothan (1978), Rendleman \& Bartter (1980), Brennan \& Schwartz (1980) models, have $\chi^{2}$ values, in excess of $5 \%$ and can be rejected at the $95 \%$ confidence level. Except for the Cox, Ingersoll \& Ross (1980), and the Cox \& Ross (1976) models, those assume $\gamma>1$. These models present values of the $\chi^{2}$ relatively low and cannot be rejected at the $5 \%$ significance level.

These results suggest that the relation between interest rate volatility and the level of $r$ is the most important feature of the dynamic model of the short term interest rate. This is significant since the Vasicek (1977) and Merton (1973) models are often criticized for allowing negative interest rates. This result indicates that a far more serious drawback of these models is their implication that interest rate changes are homoskedastic.

The estimates of the models provide also a number of interesting insights about the dynamics of the short term interest rate. First, the weak evidence of the mean reversion in the short term interest rate; the parameter $\beta$ is insignificant in the unrestricted model and also in all the restricted models. Second, the conditional volatility of the process is highly sensitive to the level of the short term interest rate. The unconstrained estimate of $\gamma$ in the Cox \& Ross (1976) and Chan, Karoly, Longstaff \& Sanders (1992) models are respectively 1.5513 and 1.5424 .

This result is important since these values are higher than the values used in most of the models. In particular, six of the eight nested models imply $0 \leq \gamma \leq 1$. The t-statistic for $\gamma$ is 9.20 and 8.05 respectively for the Cox \& Ross (1976) and Chan, Karoly, Longstaff \& Sanders (1992) models, which imply that the parameter $\gamma$ is highly significant. These results prove the importance of the relation between volatility of the interest rate and the level of the short term interest rate in the dynamic of the interest rate and that the degree of the sensitivity is higher than 1.5.

These findings are similar to those of Ferreira (1998) that has followed the same approach for Portuguese interest rates. The results show a weak mean reverting effect and a high sensitivity of the volatility to the interest rate level equal to 1.13.

The last two columns of the Table 3 present the results of the forecast power of all the models for interest rate changes and the squared interest rate changes. The first $\mathrm{R}^{2}$ measure describes the fit of the various models for the actual yield changes. Expect for the Merton (1973), Dothan (1978), Cox, Ingersoll \& Ross (1980), and the Cox \& Ross (1976) models which have no explanatory power for interest rate changes, the other models are similar in their forecast ability. They explain only $0.01 \%$ to $0.13 \%$ of the total variation in yield changes.

For the volatility of interest rate changes, the proportion of the total variation in volatility captured by the various models ranges from $0.44 \%$ from the Cox, Ingersoll \& Ross (1985) model to $17.4 \%$ for the \& Ross (1976) model. Note that the $\mathrm{R}^{2}$ for the Merton (1973) and Vasicek (1977) models are zero since these models imply that the volatility of interest rate changes is constant. Remark that the higher predictive power for the volatility for interest rate changes is for the models that assume an estimated value of $\gamma \geq 1.5$. It is equal to $17.4 \%$ for the Cox \& Ross (1976) model and $15.86 \%$ for the Chan, Karoly, Longstaff \& Schwartz (1992) model.

These results are similar to those produced by the $\chi^{2}$ test, which prove again the importance of the sensibility of the volatility of the interest rate to the level of short term 
interest rate in the dynamic of the spot interest rate. Figure 2 plot the absolute value of the interest rate changes and the estimated conditional volatility estimate from the Cox, Ingersoll \& Ross (1985) and the Chan, Karoly, Longstaff \& Schwartz (1992) models.

We remark that contrary to the Cox Ingersoll \& Ross (1985) model, the Chan, Karoly, Longstaff \& Schwartz (1992) model reproduce nearly exactly the shape of observed volatility of interest rate changes without adjusting the actual levels of interest rates .

Table 3: Estimates of alternative models for the short term interest rate

\begin{tabular}{|c|c|c|c|c|c|c|c|c|}
\hline Models & $a$ & $b$ & $\sigma^{2}$ & $\gamma$ & $\begin{array}{c}\chi^{2} \text { test } \\
(p- \\
\text { value })\end{array}$ & $d l$ & $R_{1}^{2}$ & $R_{2}^{2}$ \\
\hline $\begin{array}{l}\text { Chan Karoly } \\
\text { Longstaff } \\
\text { Sanders (1992) }\end{array}$ & $\begin{array}{l}0.004510 \\
(0.45)\end{array}$ & $\begin{array}{r}- \\
0.113786 \\
(-0.54)\end{array}$ & $\begin{array}{l}0.915860 \\
(1.55)\end{array}$ & $\begin{array}{c}\mathbf{1 . 5 4 2 4 1 6} \\
(8.05)\end{array}$ & 0.0000 & 0 & 0.0008 & 0.1586 \\
\hline Merton (1973) & $\begin{array}{c}- \\
0.000776 \\
(-0.36)\end{array}$ & 0.0000 & $\begin{array}{c}0.000121 \\
(6.05)\end{array}$ & 0.0000 & $\begin{array}{l}15.5003 \\
(0.0004)\end{array}$ & 2 & 0.0000 & 0.0000 \\
\hline Vasicek (1977) & $\begin{array}{l}0.002520 \\
(0.27)\end{array}$ & $\begin{array}{c}- \\
0.071269 \\
(-0.34)\end{array}$ & $\begin{array}{l}0.000122 \\
(5.98)\end{array}$ & 0.0000 & $\begin{array}{l}15.5172 \\
(0.0001)\end{array}$ & 1 & 0.0003 & 0.0000 \\
\hline $\begin{array}{l}\text { Cox Ingersoll } \\
\text { and Ross(1985) }\end{array}$ & $\begin{array}{c}0.003209 \\
(0.34)\end{array}$ & $\begin{array}{c}- \\
0.092045 \\
(-0.44)\end{array}$ & $\begin{array}{c}0.002739 \\
(6.91)\end{array}$ & 0.5000 & $\begin{array}{l}14.5178 \\
(0.0001)\end{array}$ & 1 & 0.0005 & 0.0044 \\
\hline Dothan (1978) & 0.0000 & 0.0000 & $\begin{array}{c}0.048578 \\
(8.52)\end{array}$ & 1.0000 & $\begin{array}{l}10.3857 \\
(0.0156)\end{array}$ & 3 & 0.0000 & 0.0298 \\
\hline $\begin{array}{l}\text { Rendleman and } \\
\text { Bartter (1980) }\end{array}$ & 0.0000 & $\begin{array}{c}0.028005 \\
(-0.62)\end{array}$ & $\begin{array}{c}0.049714 \\
(8.31)\end{array}$ & 1.0000 & $\begin{array}{l}10.0911 \\
(0.0064)\end{array}$ & 2 & 0.0001 & 0.0312 \\
\hline $\begin{array}{l}\text { Brennan and } \\
\text { Schwartz } \\
(1980)\end{array}$ & $\begin{array}{c}0.005209 \\
(0.55)\end{array}$ & $\begin{array}{c}- \\
0.141874 \\
(-0.67)\end{array}$ & $\begin{array}{c}0.050287 \\
(8.25)\end{array}$ & 1.0000 & $\begin{array}{c}9.8074 \\
(0.0017)\end{array}$ & 1 & 0.0013 & 0.0319 \\
\hline $\begin{array}{l}\text { Cox Ingersoll } \\
\text { and Ross(1980) }\end{array}$ & 0.0000 & 0.0000 & $\begin{array}{c}0.725366 \\
(9.69)\end{array}$ & 1.5000 & $\begin{array}{c}0.5475 \\
(0.9083)\end{array}$ & 3 & 0.0000 & 0.1379 \\
\hline $\begin{array}{l}\text { Cox and Ross } \\
\text { (1976) }\end{array}$ & 0.0000 & $\begin{array}{c}0.015935 \\
(-0.35)\end{array}$ & $\begin{array}{c}0.949175 \\
\quad(1.57)\end{array}$ & $\begin{array}{c}\mathbf{1 . 5 5 1 2 9 5} \\
(8.20)\end{array}$ & $\begin{array}{c}0.2243 \\
(0.6358)\end{array}$ & 1 & 0.0000 & 0.174 \\
\hline
\end{tabular}




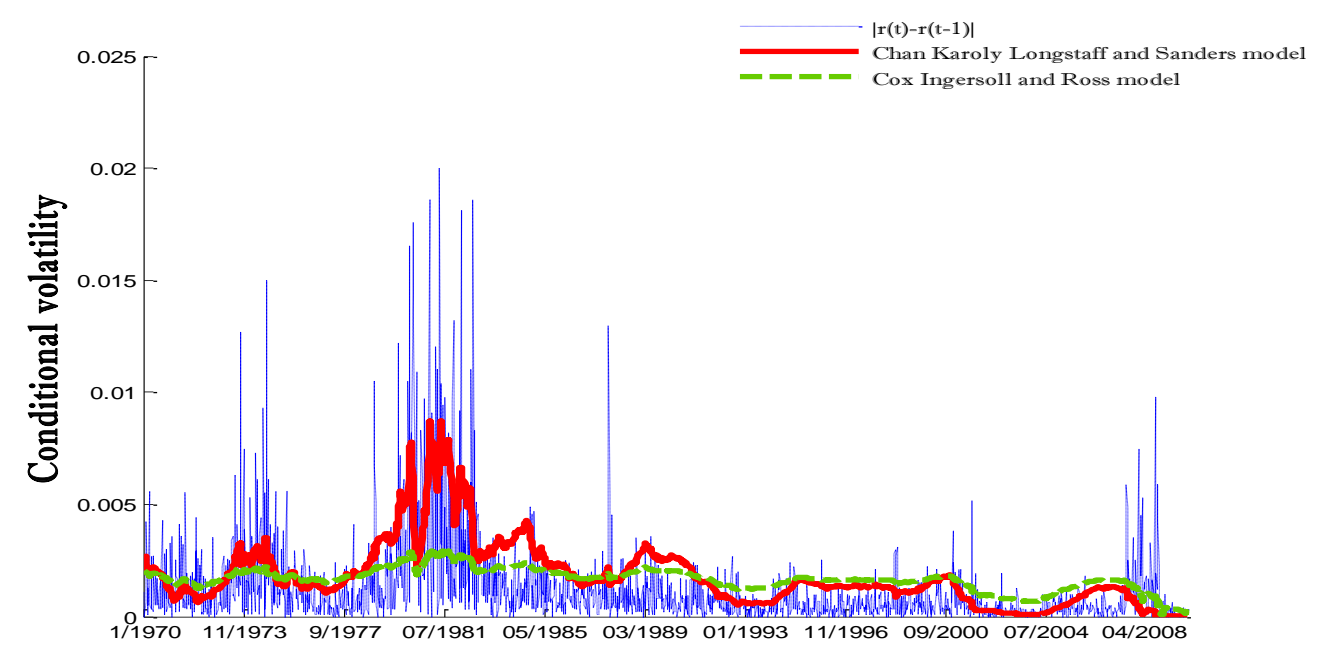

Figure 2: Forecast of weekly ex post volatility of short term interest rate using the Cox, Ingersoll \& Ross (1985) and the Chan, Karoly, Longstaff \& Schwartz (1992) models.

\section{2. Estimation Results of Nonlinear Model}

From the table 4, we note first that all the conditions imposed by Ait Sahalia (1996b) are respected. Second, all parameters except $\beta_{3}$ are not significant. The mean reverting parameter $\alpha_{2}$, is not significant as proved for linear models.

The figure 3 plots the nonlinear drift of the Ait Sahalia (1996b) model. We note that for the central area of the interest rate between $1 \%$ and $18 \%$, the average is almost zero. In addition for interest rates below $1 \%$, the nonlinearity of the drift strongly pushing interest rates to the mean area. Or since 2008, the short term interest rates show low values converge to almost zero and the short term interest rate has not recorded a mean reversion as stipulated by Ait Sahalia (1996b) model. In addition according to the model of Ait Sahalia (1996b), the mean reversion effect is manifested at very high interest rates above $20 \%$. But it is almost impossible to achieve these values for the short term interest rates.

Table 4: The parameters estimates of the Ait Sahalia (1966b) model

\begin{tabular}{|l|c|c|c|}
\hline Parameters & Estimated values & standard erreur & t-stat \\
\hline$\alpha_{0}$ & $1,64 \mathrm{e}^{-4}$ & 0,2086 & 0,00078 \\
\hline$\alpha_{1}$ & 0,019 & 2,1332 & 0,0089 \\
\hline$\alpha_{2}$ & -0.1258 & 7,2240 & 0,0174 \\
\hline$\alpha_{3}$ & $1,154 \mathrm{e}^{-5}$ & 0,0068 & 0,0022 \\
\hline$\beta_{0}$ & $8,8 \mathrm{e}^{-6}$ & 0,0001 & 0,088 \\
\hline$\beta_{1}$ & 0,0026 & 0,0017 & 0,1529 \\
\hline$\beta_{2}$ & 0,0238 & 0,0165 & 1,4424 \\
\hline$\beta_{3}$ & 2,0319 & 0,5775 & 3,5184 \\
\hline
\end{tabular}


The figure 3 plots the nonlinear drift of the Ait Sahalia (1996b) model. We note that for the central area of the interest rate between $1 \%$ and $18 \%$, the average is almost zero. In addition for interest rates below $1 \%$, the nonlinearity of the drift strongly pushing interest rates to the mean area. Or since 2008, the short term interest rates show low values converge to almost zero and the short term interest rate has not recorded a mean reversion as stipulated by Ait Sahalia (1996b) model. In addition according to the model of Ait Sahalia (1996b), the mean reversion effect is manifested at very high interest rates above $20 \%$. But it is almost impossible to achieve these values for the short term interest rates.

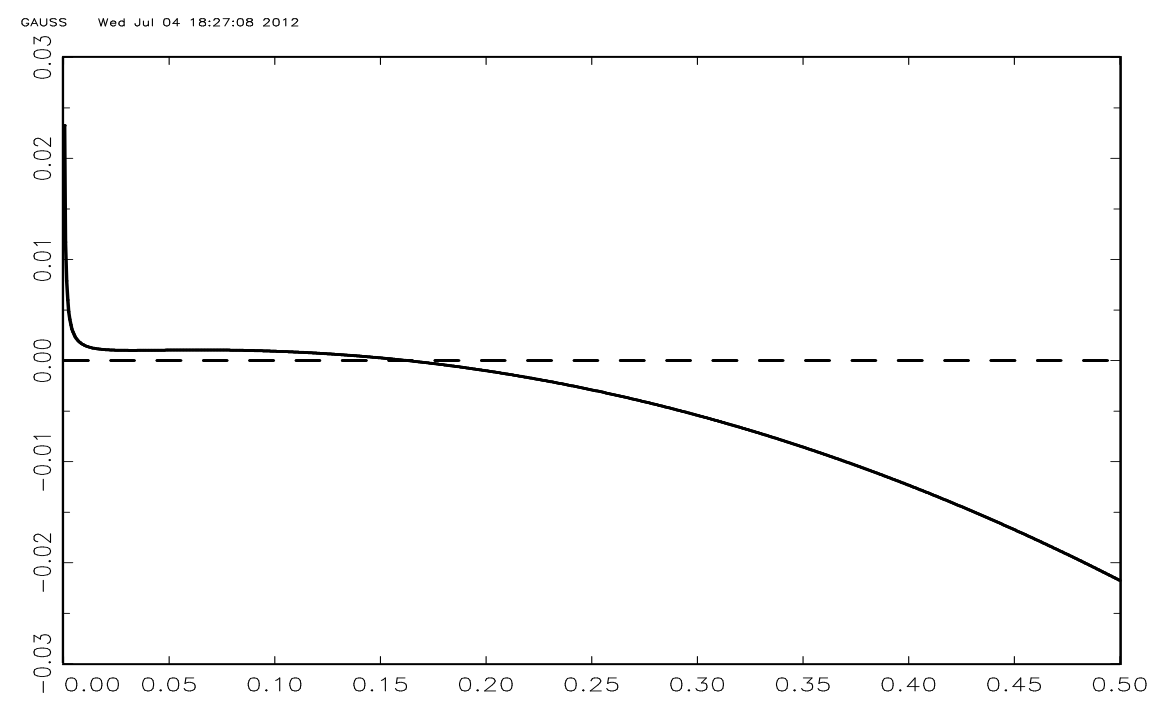

Figure 3: The nonlinear drift of the Ait Sahalia (1996b) model

The figure 4 shows the nonlinear diffusion of the Ait Sahalia model (1996b). We note that volatility increases with the level of interest rates. This confirms the presence of the effect level in the diffusion function.

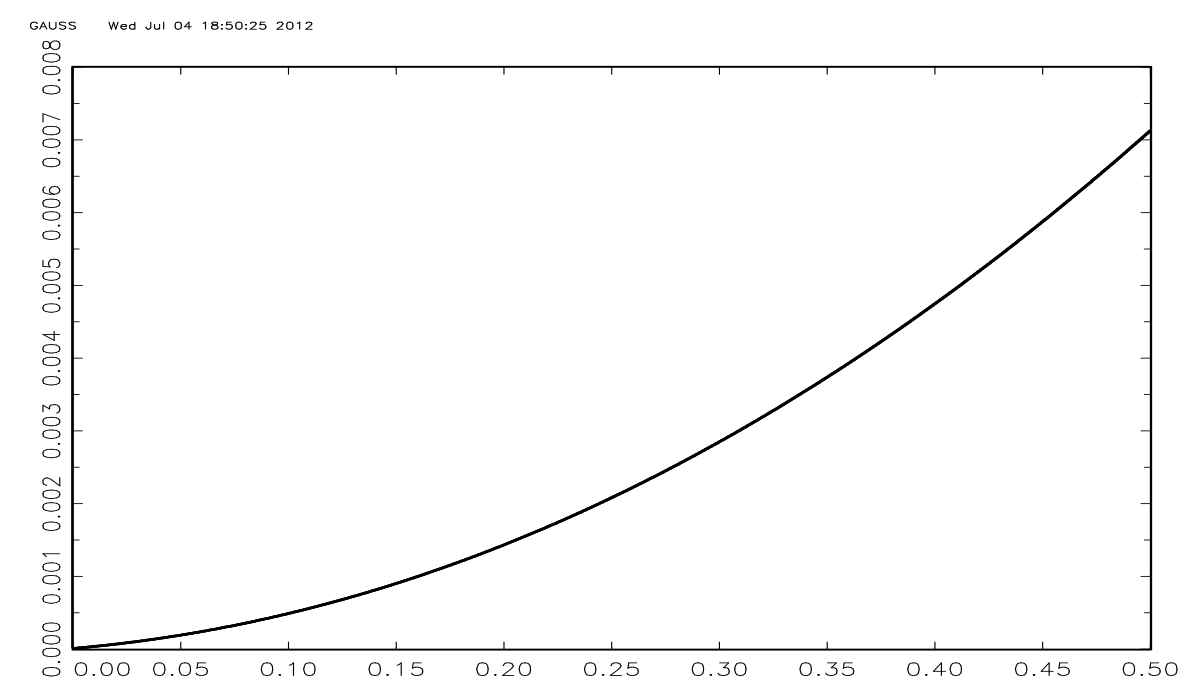

Figure 4: The non linear volatility of the Ait Sahalia (1996b) interest rate model 


\subsection{Monte Carlo Simulation Results}

The stochastic path of the interest rate produced by the Ait Sahalia model (1996b) is compared to those produced by the linear models of Chan, Karoly, Longstaff \& Schwartz (CKLS, 1992), of Cox \& Ross (CEV, 1976), of Brennan \& Schwartz (BS, 1980) and Rendleman and Bartter (GBM, 1980).

These models are firstly the best linear short-term interest rates models among the studied models. On the other hand they present the main characteristics of the stochastic process of short-term interest rate, ie the mean reversion effect and the elasticity of volatility. The objective of this choice is to compare the contribution of nonlinear parameterization of Ait Sahalia (1996b) compared to those presented by these linear models in the estimation of the real short interest rates term stochastic path.

The simulation periods considered are the three in the sample periods "1979-1982", "1997-1998", "2007-2008" and the out of the sample period "2010-2011".

From figure 5, we see that as for the linear models, the path of short term interest rates generated by the nonlinear model of Ait Sahalia (1996b) does not adequately reproduce the real interest rate path and this for the four periods studied.

The table 5 shows the mean square error for the different models.

Table 5: Mean Squared Error of the Monte Carlo simulation

\begin{tabular}{|c|c|c|c|c|}
\hline \multirow{2}{*}{ Models } & \multicolumn{4}{|c|}{ MSE } \\
\hline & $\begin{array}{c}\text { "In sample » } \\
10701082 \text { ? }\end{array}$ & $\begin{array}{c}\text { «In sample » } \\
10071008\end{array}$ & 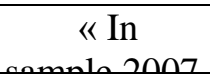 & $\begin{array}{c}\text { "out of sample » } \\
20102011\end{array}$ \\
\hline CKLS & $4.5662 \mathrm{e}-09$ & $4.6321 \mathrm{e}-007$ & $2.3690 \mathrm{e}-005$ & $2.1168 \mathrm{e}-007$ \\
\hline $\mathrm{CEV}$ & $6.4781 \mathrm{e}-008$ & $4.4038 \mathrm{e}-008$ & $1.9411 \mathrm{e}-005$ & $2.4801 \mathrm{e}-009$ \\
\hline GBM & $6.8501 \mathrm{e}-007$ & $1.5526 \mathrm{e}-009$ & $1.7262 \mathrm{e}-005$ & 2.1617e-009 \\
\hline BS & $1.2494 \mathrm{e}-006$ & $3.0099 \mathrm{e}-009$ & $1.7230 \mathrm{e}-005$ & $2.2512 \mathrm{e}-007$ \\
\hline Ait & $1.0797 \mathrm{e}-006$ & $5.6118 \mathrm{e}-007$ & $2.4278 \mathrm{e}-005$ & $3.0300 \mathrm{e}-007$ \\
\hline
\end{tabular}



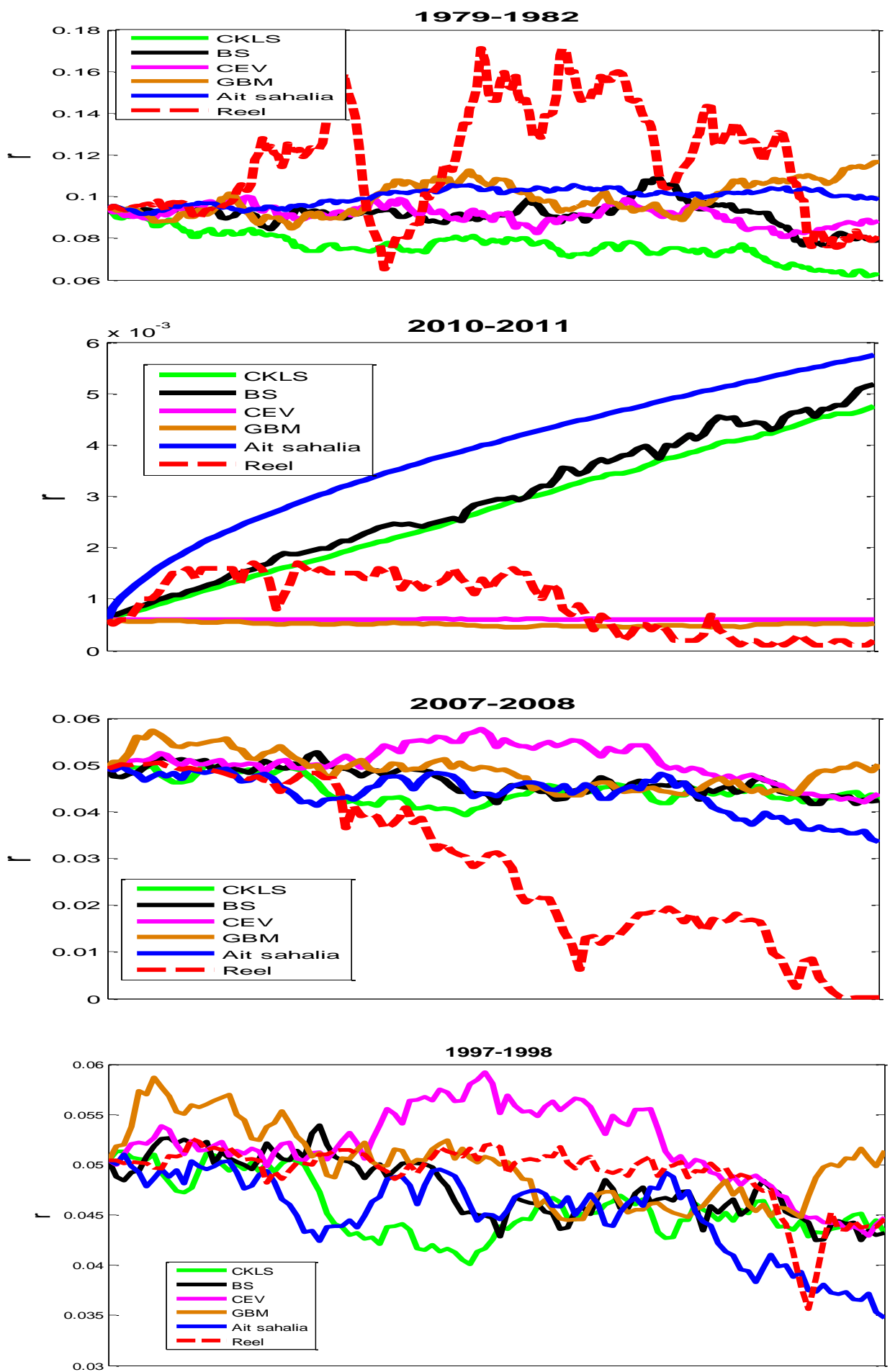

Figure 5: (a) Simulation « out of the sample » 2010-2011 period, simulation "in the sample"; (b) 1979-1982 period; (c) 2007-2008 period, (d) 1997-1998 period. 


\subsection{Estimation Results of the Nonparametric Model}

To estimate the drift and the diffusion functions, the stationary density of the short term interest rate is estimated first and plotted in Figure 6. The nonparametric stationary density is obviously not normal with a flatter right tail than the normal density.

From Figure 7, we note that the drift is constant and it is close to being zero for low and medium values of interest rate. But when the short rate is beyond $14 \%$; the short rate drift decrease dramatically. It presents a negative linear trend. This confirm the empirical finding of Ait Sahalia (1996a), Stanton (1997), Jiang and Knight (1997), Jiang (1998), Sam and Jiang (2009) and Gospodinov and Hirukawa (2011) that the drift term of the short term interest rate is zero for the most of interest rate ranges and overall nonlinearly mean reverting.

These results prove also the finding of Arapis and Gao (2006) that the nonparametric drift is unlike the linear mean reverting specification.

These findings suggest that interest rates follow a random walk at low and medium level, while being overall stationary. But because of the high level of interest rates (beyond $14 \%$ ) observed mainly during the period of early 80 's, the nonstationarity test based on the short term interest rate level is rejected.

Compared to the linear mean reverting drift function, the nonparametric drift function shows a much weaker mean reverting property for low and medium interest rate but a much stronger mean reverting property for high interest rates. The mean reverting effect occurs only when the rate of interest is almost over $14 \%$. This finding confirms that the mean reverting is not very significant especially for low and middle levels of the Interest rates.

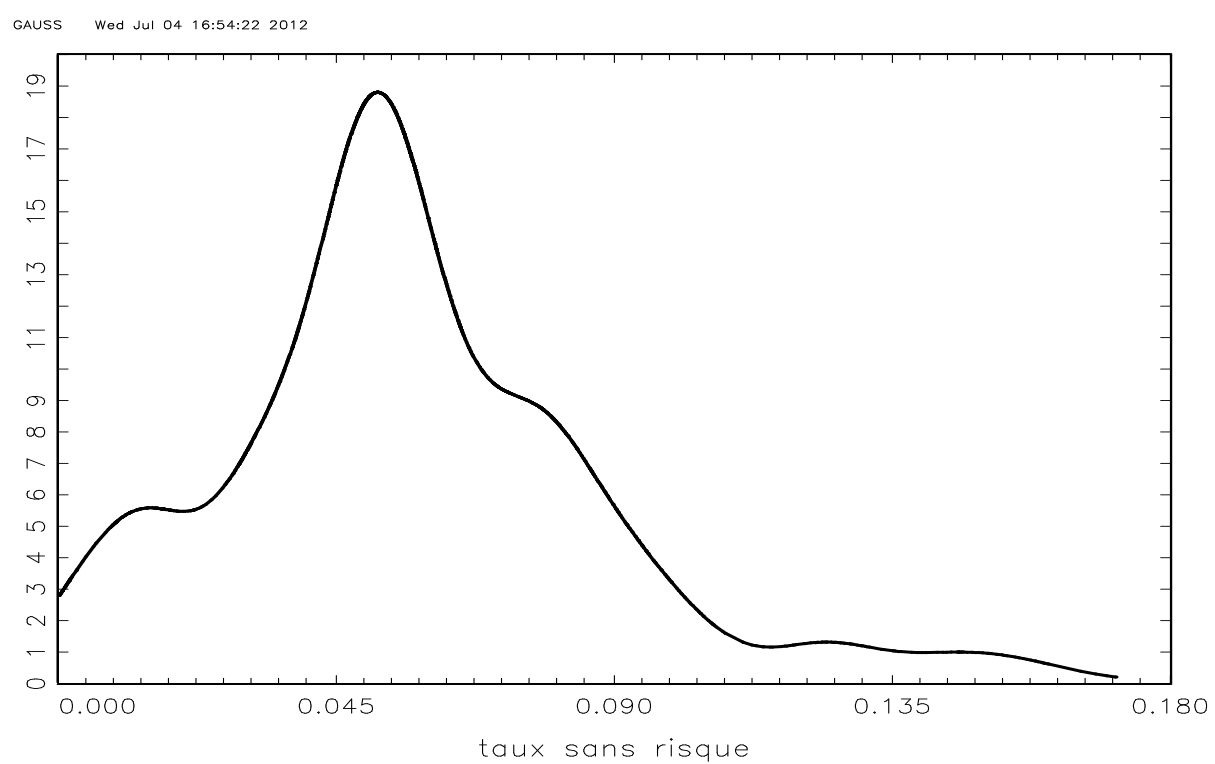

Figure 6: Nonparametric stationary density of short rate 


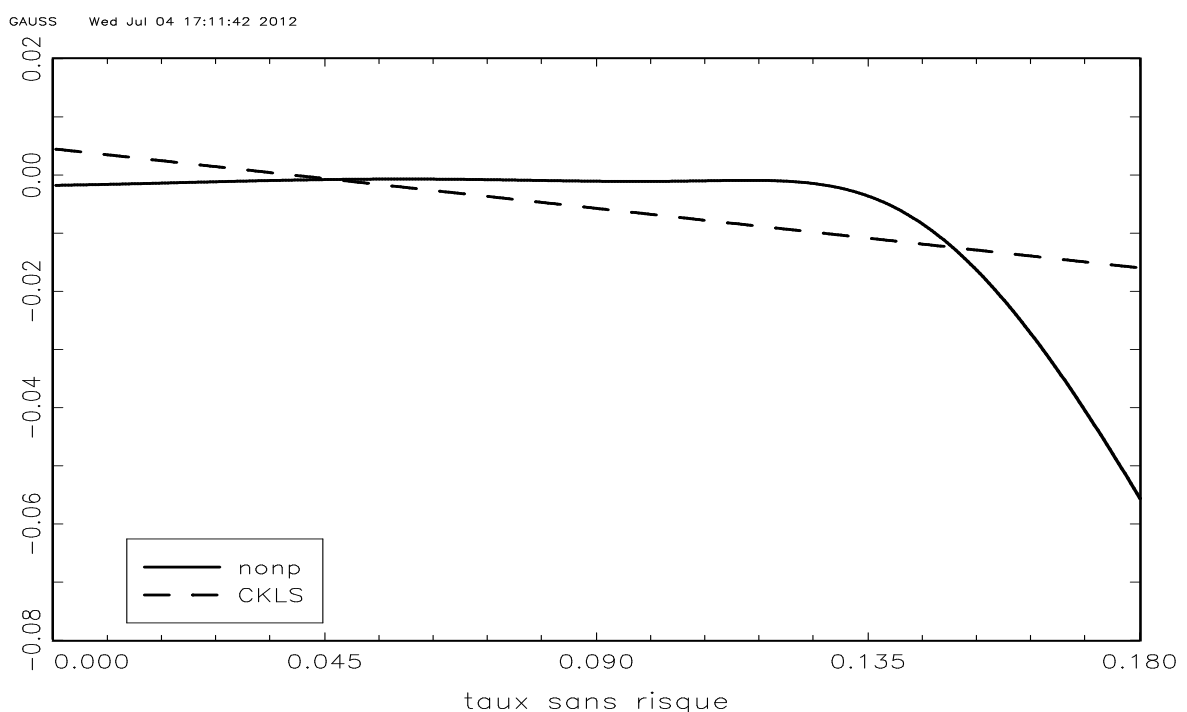

Figure 7: Nonparametric and Chan, Karoly, Longstaff \& Schwartz (CKLS) drift functions

Compared to the nonlinear drift function (figure 8), we note that they are different. Indeed nonparametric drift presents a mean reverting effect for interest rate values greater than $14 \%$ whereas for such values, the drift of the Ait Sahalia (1996b) model is almost zero and the mean reverting effect occurs only for interest rate values greater than $20 \%$.

We deduce that the nonlinear drift specification proposed by Ait Sahalia (1996b) does not reproduce the drift of the observed data, given by the nonparametric estimator.

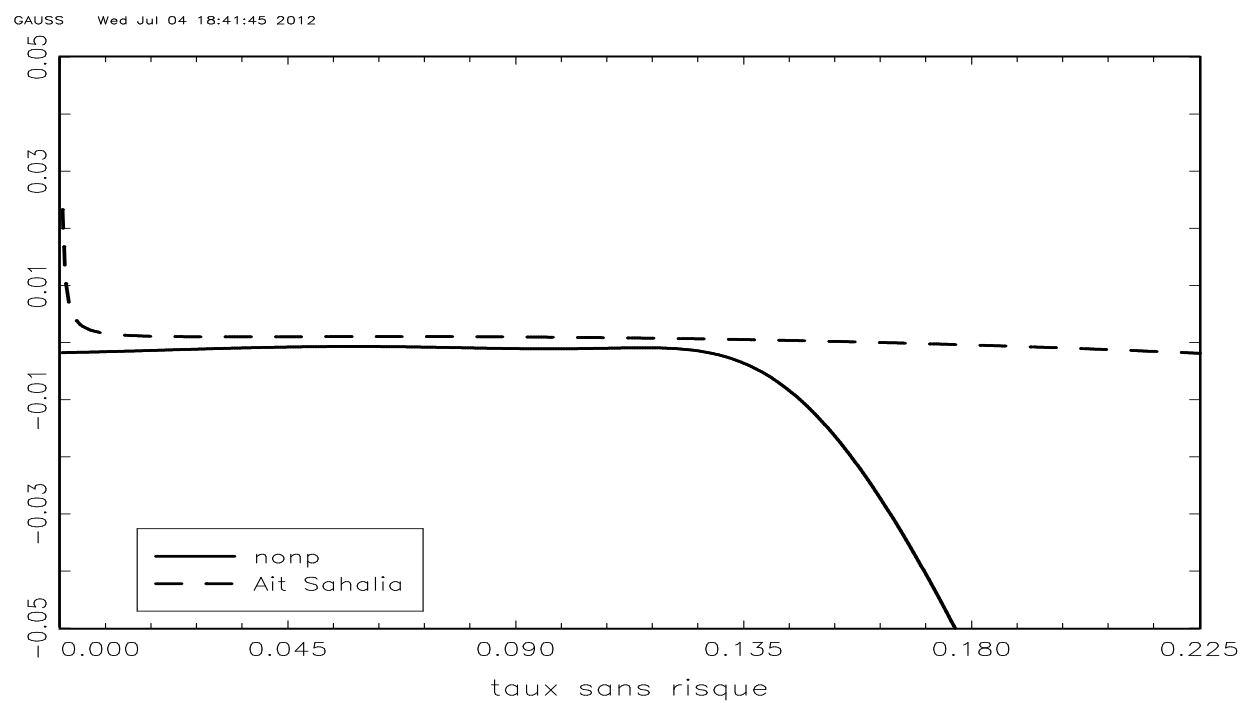

Figure 8: Nonparametric and the nonlinear drift functions

The nonparametric diffusion function is plotted in Figure 9 and compared to those of the Chan, Karoly, Longstaff \& Schwartz (CKLS, 1992), Cox, Ingersoll and Ross (CIR-SR, 1985 ) and Ait Sahalia models (1996b). Noticeable features of the nonparametric diffusion function estimator include: first, the diffusion function is a nonlinear but overall 
increasing function of the short rate, supporting the "level-effect" conjecture and rejecting the constant volatility model. That is, low interest rates are associated with low interest rates volatility and high interest rates are associated with high interest rates volatility. This result proves that the volatility is nonlinear contrary to the parametric models that suppose that the volatility is linear. These results are similar to those of Ait Sahalia (1996a), Stanton (1997), Jiang and Knight (1997), Jiang (1998), Sam and Jiang (2009) and Gospodinov and Hirukawa (2011).

The nonlinear diffusion of Ait Sahalia (1996b) model is very different from the nonparametric one, it is very low and the level effect is also very low.

We deduce that, as for the drift the diffusion specification of the Ait Sahalia (1996b) model does not reproduce the diffusion of the observed data.

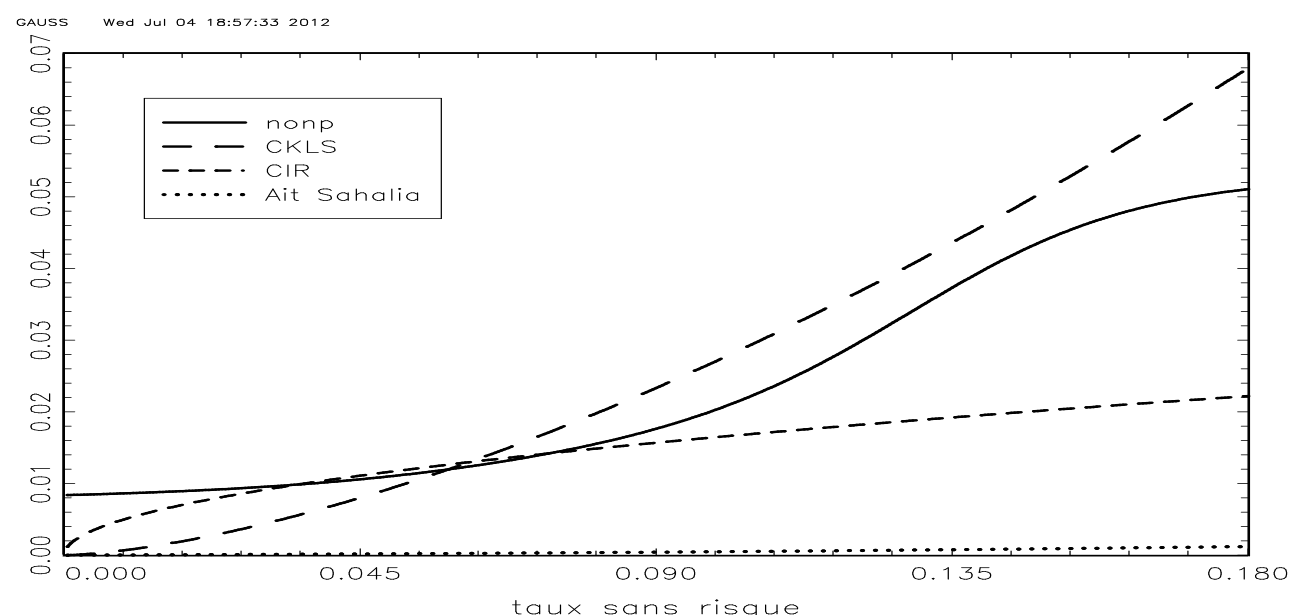

Figure 9: Non-parametric, nonlinear, CKLS and CIR-SR diffusion functions

\section{Conclusion}

In this paper, we investigate the appropriate features of the short term interest rate stochastic process. The results indicated that models that allowed the variability of interest rates to depend upon the level of interest rate captured the dynamic behaviour of short term interest rates more successfully. The level effect is such that the interest rate volatility is positively correlated with the level of interest rates. In addition the results prove also that the evidence on mean reversion in the short term to be not inconclusive.

The nonparametric estimation of the drift and the diffusion functions of the short term interest rate process prove also the importance of the "level effect" in the interest rate volatility and that the "mean reversion " effect is significant only for very high level of interest rates. The drift and diffusion functions appear also to be nonlinear.

The results show also that the Ait Sahalia (1996b) nonlinear model is unable to predict the real stochastic path of the short-term interest rates process.

The problem is in the specification of the drift and the diffusion functions proposed by Ait Sahalia (1996b) model.

These results lead us to question on the appropriate nonlinear specification of the short term interest rate model. 


\section{References}

[1] Ait-sahalia, Y (1996a), Nonparametric pricing of interest rate derivative securities, Econometrica 64, 527-560.

[2] Ait-sahalia, Y (1996b), Testing continuous-time models of the spot interest rate, Review of Financial Studies 9, 385-426.

[3] Ait-Sahalia, Y (2002), Maximum likelihood estimation of discretly sampled diffusions: a closed form approximation approach, Econometrica 70, 1, 223-262.

[4] Arapis, M. Gao, J (2006), Empirical comparisons in short-term interest rate models using nonparametric methods. Journal of Financial Econometrics, 4, 310-345.

[5] Bandi, F. Phillips, P. (2003), Fully nonparametric estimation of scalar diffusion models. Econometrica 71 (1), 241-283.

[6] Brennan, M., E. Schwartz (1979), A Continuous Time Approach to the Pricing of Bonds, Journal of Banking and Finance, 3, 135-155.

[7] Chan, K. C., G A Karolyi, F. A. Lonstaff, A. B. Sanders (1992), An empirical comparison of alternative models of the short-term interest rate, Journal of Finance 47, 1209-1227.

[8] Cox, J. C., J. E. Ingersoll, S. A. Ross (1980), An Analysis of Variable Rate Loan Contracts. Journal of Finance 35, 389-403.

[9] Cox, J. C., J. E. Ingersoll, S. A. Ross (1985a), An intertemporal general equilibrium model of asset prices, Econometrica, 53, 363-384

[10] Cox, J. C., J. E. Ingersoll, S. A. Ross (1985b), A Theory of the Term Structure of Interest Rates, Econometrica, 53, 385-407.

[11] Ferreira, A.M (1998), An empirical test of short term interest rate models, Review of financial markets, 1, 29- 48.

[12] Florens-Zmirou, D. (1993), On estimating the diffusion coefficient from discrete observations, Journal of Applied Probability 30, 790-804.

[11] Gospodinov, N. Hirukawa, M (2011), Nonparametric estimation of scalar diffusion processes of interest rates using asymmetric Kernels, working paper.

[12] Hansen, L. (1982), Large sample properties of generalized method of moment estimators, Econometrica, 50, 1029-1056.

[13] Jiang, G. J. Knight (1997), A nonparametric approach to the estimation of diffusion processes, with an application to a short-term interest rate model. Econometric Theory 13, 615-645.

[14] Jiang, G. J (1998), Nonparametric modeling of US interest rate term structure dynamics and the implications on the prices of derivatives securities, Journal of financial and quantitative analysis, 4, 565-499.

[15] Merton, R. (1973), Theory of Rational Option Pricing, Bell Journal of Economics and Management Science, 4, 637-654.

[16] Rendleman, R. and B, Bartter (1980), The Pricing of Options on Debt Securities. Journal of Financial and Quantitative Analysis, 15, 11-24.

[17] Renò, R. Roma, A. Stephen, S (2006), A Comparison of Alternative Nonparametric estimators of the Short Rate Diffusion Coefficient, Economic Notes, $35,227-252$.

[18] Sam, A G. Jiang, G (2009), Nonparametric estimation of the short rate diffusion process from a panel of yields, Journal of Financial and Quantitative Analysis, 44, 1197-1230. 
[19] Stanton, R. (1997), A Nonparametric Model of Term Structure Dynamics and the Market Price of Interest Rate Risk, Journal of Finance, 52, 1973-2002.

[20] Vasicek, O. A. (1977), An equilibrium characterization of the term structure, Journal of Financial Economics 5, 177-188. 